\title{
Aneurisma tóraco-abdominal inflamatório
}

\author{
Inflammatory thoracoabdominal aneurysm
}

\author{
Eduardo Faccini Rocha ${ }^{1}$, George C archedi Luccas ${ }^{2}$, Luis Baldini N eto ${ }^{3}$
}

\begin{abstract}
Resumo
O s autores relatam o caso de um paciente apresentando aneurisma tóraco-abdominal tipo IV de etiologia inflamatória. Esse paciente foi submetido à correção cirúrgica eletiva através de incisão tóraco-abdominal, rotação medial das vísceras, com dificuldade, devido às aderências, eutilização de perfusão visceral duranteo pinçamento supracelíaco, com um circuito de circulação extracorpórea modificado.
\end{abstract}

Palavras-chave: aneurisma da aorta abdominal, inflamação, aneurismas da aorta torácica.

D esde o primeiro reparo com sucesso de um aneurisma deaortaabdominal, feito por $C$ harles D ubost, em 29 demarço de 1951, com enxerto homólogo, a cirurgia eletiva desses aneurismas tornou-se um procedimento de rotina, com morbidade e mortalidade relativamente baixas $^{1}$. Alguns fatores, entretanto, podem aumentar consideravel mentea complexidadeda cirurgiadosaneurismas. Entre eles, podemos citar os aneurismas tóracoabdominais (ATA), para-renais, micóticos ou infecciosos, inflamatórios, reoperações, anomalias venosas associadas ${ }^{2}$ e outros.

A ocorrência de um aneurisma inflamatório envolvendo a aorta tóraco-abdominal é incomum ${ }^{3,4}$. $\mathrm{N}$ esses casos, a necessidade de pinçamento supracelíaco, associada às múltiplas aderências do processo inflamatório, tornam a cirurgia um grande desafio à equipe vascular.

1. Médico contratado, Disciplina de Cirurgia Vascular Periférica, Faculdade de Ciências Médicas, Universidade Estadual de Campinas (UNICAMP), Campinas, SP.

2. Livre-docente. Professor, Disciplina de Cirurgia Vascular Periférica, Faculdade de Ciências Médicas, UNICAMP, Campinas, SP.

3. Residente de Cirurgia Vascular, Faculdade de Ciências Médicas, UNICAMP, Campinas, SP.

Artigo submetido em 29.03.05, aceito em 12.08.05.

\begin{abstract}
The authors report an unusual case of a patient with a type IV thoracoabdominal inflammatory aneurysm. This patient was submitted to surgery through a thoracoabdominal incision, medial visceral rotation with difficulty due the adherences and visceral perfusion during the supraceliac clamping with a modified assisted circulatory device.
\end{abstract}

Key words: abdominal aortic aneurysm, inflammation, thoracic aortic aneurysm.

\section{Relato do caso}

E.H., 65 anos, sexo masculino, encaminhado ao Ambulatório de Cirurgia Vascular do Hospital das Clínicas da U N ICAM P, queixando-se de desconforto abdominal há 2 meses. A presentava como antecedentes: tabagismo, insuficiência renal crônica decausa pósrenal em acompanhamento com a nefrologia.

Ao exame físico, encontrava-se em bom estado geral, afebril, com pressão arterial de $130 / 80 \mathrm{mmH}$ g, massa pulsátil indolor em mesogástrio com cerca de $6 \mathrm{~cm}$ de diâmetro e sinal de DeBabey presente. Todos os pulsos estavam presentes e sem sopros.

A tomografia evidenciava um aneurisma com início na emergência da artéria mesentérica superior e maior diâmetro de $8 \mathrm{~cm}$ abaixo das renais, com sinais de espessamento de sua paredeem toda circunferência (Figura 1).

$\mathrm{N}$ a avaliação pré-operatória, o risco cardiológico foi estimado em 10\% (G oldman II): ecocardiograma evidenciando insuficiências mitral e aórtica leves, fração de ejeção de 58,87\%, além da avaliação pulmonar, que identificou al to risco para complicações (VEF1 de $112 \%$ epO $_{2}$ de $71,2 \mathrm{mmH} \mathrm{g}_{1} \mathrm{pCO}_{2}$ de 36 $\mathrm{mmH} g$ e saturação de $\mathrm{O}_{2}$ de $96 \%$ ). Apresentava creatinina de $1,9 \mathrm{mg} / \mathrm{dl}$. 


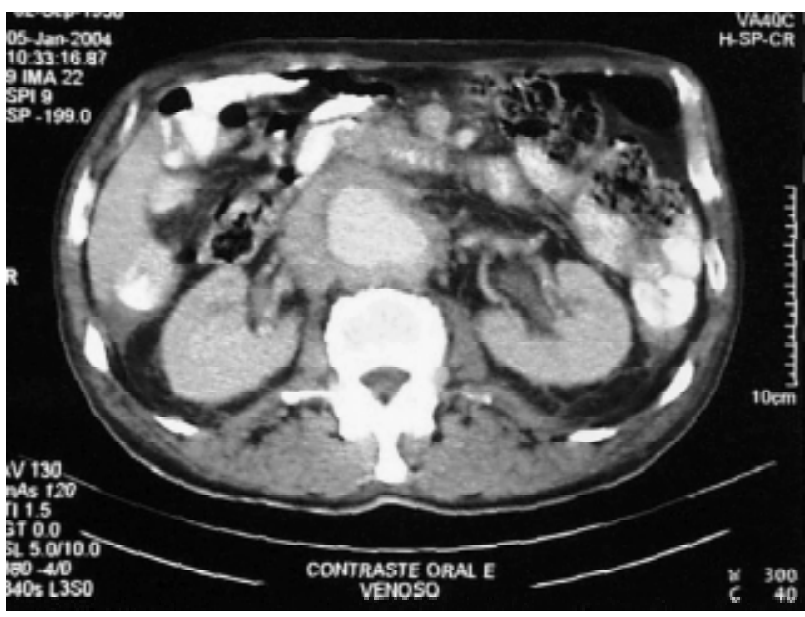

Figura 1 - Aneurisma na altura da saída das artérias renais com espessamento da parede da aorta

Foi indicada a correção cirúrgica com monitoração com cateter deSwan-G anz, acesso venoso central, pressão arterial média em radial direita, intubação orotraqueal seletiva e utilização de dispositivo para recuperação sanguínea intra-operatória (C ats da F resenius).

0 paciente foi posicionado em decúbito lateral direito, com rotação escapular de 70 graus e pélvica de 30 graus. A abordagem e o reparo da veia femoral comum direita foram realizados para posterior cateterização para o circuito de circulação extracorpórea modificado (CCEM ), que consistia na associação de um circuito de circulação extracorpórea com uma bomba de infusão venosa rápida. N este caso, não utilizamos o reservatório de cardiotomia na linha arterial. 0 reservatório foi utilizado para infusão venosa de Ringer lactato. T ambém não foram utilizados filtros ou trocadores de calor, e utilizou-se oxigenador de neonatologia, tudo isso visando diminuir a dose de heparina necessária nesse circuito (tempo de coagulação ativada desejado de 300 s), não diferindo muito da heparinização utilizada com a técnica clamp and go. 0 fluxo necessário para perfusão visceral é de, aproximadamente, $60 \mathrm{ml} / \mathrm{min}$ por artéria, e o oxigenador de neonatologia tem capacidade para até $1.000 \mathrm{ml} / \mathrm{min}$.

O utra grande vantagem do circuito é a infusão venosa rápida através da Biopump, com o shunt associado ao circuito. Desse modo, obteve-se uma bomba venosa com custos extras insignificantes (três conexões em Y e um reservatório). Essa infusão de volume pela bomba coincide exatamente com o final das anastomoses proximal e distal, quando se faz necessário um volume extra, devido à hipotensão que se seguirá à retirada das pinças (Figuras 2 e 3 ).

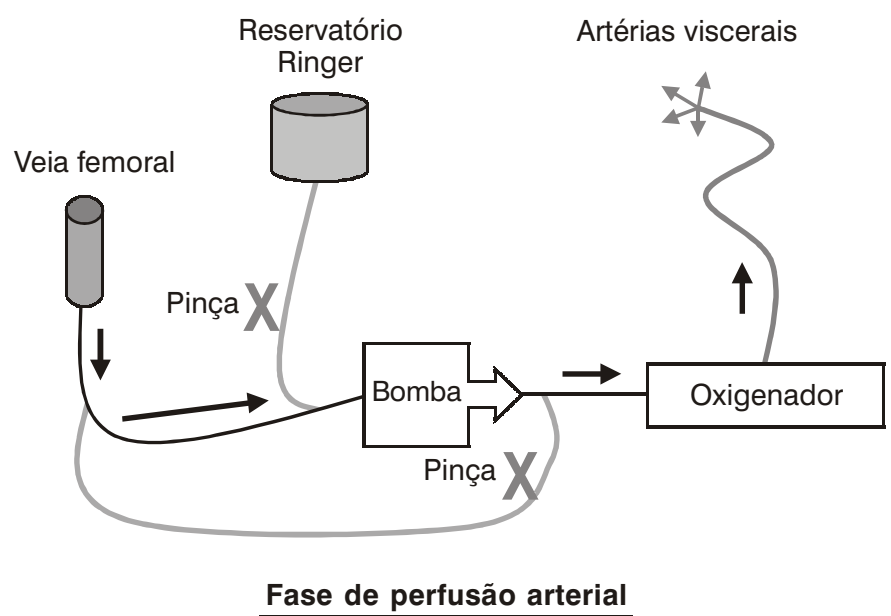

Figura 2 - Circuito circulação extracorpórea modificado, com perfusão seletiva dos troncos viscerais. $N$ ote que 0 circuito de infusão venosa está pinçado, permitindo apenas fluxo para as vísceras 


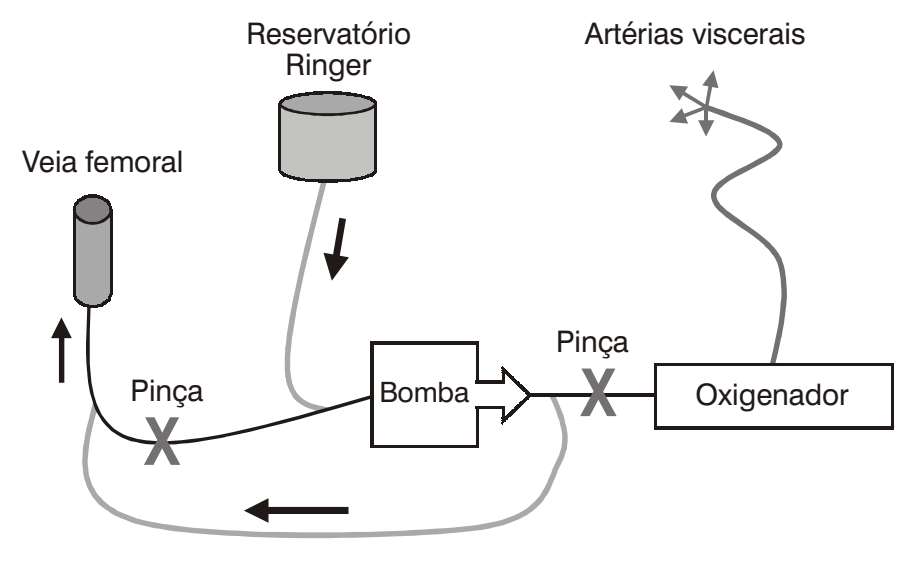

Fase de infusão venosa rápida

Figura 3 - Circuito decirculação extracorpórea modificado, com pinçamento da via de perfusão seletiva dos troncos viscerais. $N$ ote que o circuito de infusão venosa está aberto, permitindo infusão de Ringer para a veia femoral

Realizada, a seguir, a toracofrenolaparotomia, iniciando-se no nono espaço intercostal esquerdo nalinha axilar média, prolongando-se até a linha média no abdome, com extensão até a sínfise púbica. Foi feita abertura parcial do diafragma, com incisão semicircular erotação medial dasvísceras, com dificuldade, em razão do extenso processo inflamatório esangramento difuso do retroperitôneo. Foi, assim, exposta a aorta, desde a transição tóraco-abdominal atéilíacas, havendo intensa atividade inflamatória com aspecto de "porcelana", acometen do todaaaorta abdominal, além deaderências importantes dos ureteres (Figura 4).

Realizou-se reparo proximal acima do tronco celíaco e distal em artérias ilíacas. Após heparinização sistêmica com tempo de coagulação ativada (TCA) em torno de 300 segundos, foi introduzida a cânula venosa aramada (T ecnobio Ind. Com. Repr. Ltda.) de $20 \mathrm{~F}$ na veiafemoral comum direita, atéal cançar a cava inferior, sendo conectada ao CCEM .

A seguir, foi feito pinçamento proximal e distal, abertura do aneurisma (havia capa espessa de $2-3 \mathrm{~cm}$ ) desde o tronco celíaco até a bifurcação aórtica. Foram introduzidos os cateteres Pruitt de $9 \mathrm{~F}$ nos óstios visce- rais, e iniciada a perfusão com sangue oxigenado normotérmico, mantendo-se uma pressão de perfusão de $60 \mathrm{mmH} \mathrm{g}$ (fluxo total entre 300-350 ml/min).

Foi feitaainterposição deprótesedeD acron $24 \mathrm{~mm} /$ $30 \mathrm{~cm}$ (H emashield G old W oven double velour) aortoaórtica, com anastomose proximal em bisel envolvendo as artérias renal direita, mesentérica superior e tronco celíaco. A renal esquerda foi reimplantada separadamente na prótese. Ao final da anastomose proximal, os cateteres de perfusão visceral foram retirados, einiciouse infusão rápida de volume via CCEM .

Antes de soltar as pinças distais, foi feita nova infusão rápida de cristalóide, via CCEM , para evitar hipotensão. Com o paciente estável hemodinamicamente, foi retirada a cânula da veia femoral, revertida a heparina com protamina e iniciado o fechamento das cavidades torácica e abdominal.

0 tempo de isquemia visceral foi de 60 minutos para a renal esquerda e 40 minutos para as demais artérias viscerais. 0 volume de cristalóide injetado pela CCEM foi de $3.800 \mathrm{ml}$.

0 paciente permaneceu na UTI por 4 dias, tendo sido extubado e permanecendo sem drogas vasoativas 


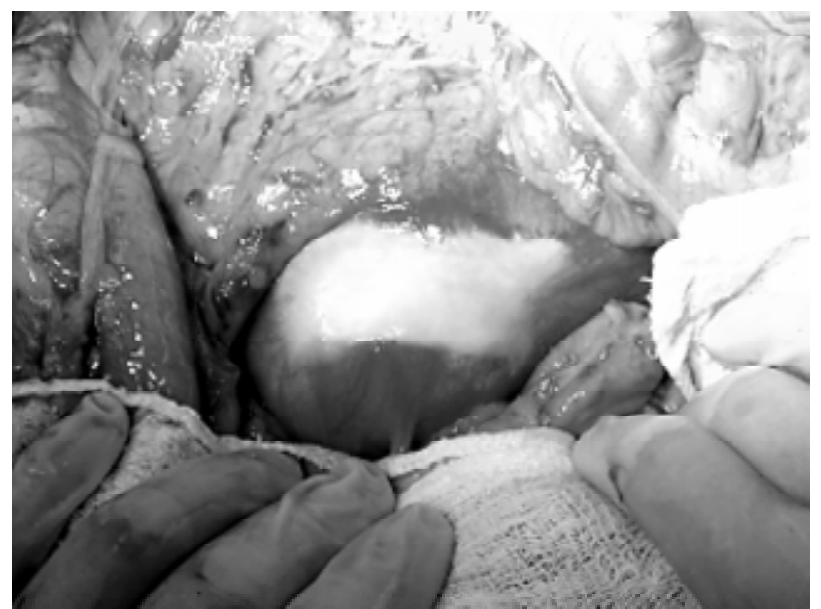

Figura 4 - Aspecto em "porcelana" da porção infra-renal do aneurisma tóraco-abdominal antes da rotação medial das vísceras

desde o primeiro dia pós-operatório, com creatinina de $1,7 \mathrm{mg} / \mathrm{dl}$. A dieta foi introduzida no terceiro dia, efoi de alta em boas condições apenas após 15 dias, devido ao dreno torácico com débito elevado e pneumonia.

\section{D iscussão}

É creditado a D ubost, em 1951, a primeira correção bem sucedida de um aneurisma de aorta abdominal, e a Etheredge, em 1954, a primeira cirurgia de um AT A ${ }^{1,5,6}$. Apesar dos inúmeros avanços médicos e técnicos, os desafios do cirurgião vascular frente a essas doenças ainda são consideráveis, especialmente em relação às complicações pós-operatórias, incluindo paraplegia, insuficiência renal, isquemia do miocárdio, complicações respiratórias, distúrbios da coagulação e outras 6,7 .

U m estudo sobrea história natural da evolução dos AT A revela que apenas $24 \%$ dos pacientes tratados conservadoramente estarão vivos após 2 anos do diagnóstico da doença, sendo que $50 \%$ das mortes são por ruptura do aneurisma ${ }^{1,5}$.

A incidência dos ATA é em torno de 5-10\% entre os aneurismas da aorta, e o AT A tipo IV representa 19 a $23 \%$ dos ATA ${ }^{1,8,9}$. Entre os ATA, o tipo IV de $C$ rawford (que envolve apenas a aorta abdominal, in- cluindo os troncos viscerais) é o que apresenta menor risco de complicações intra- e pós-operatórias. Entretanto, quando comparado à correção dos aneurismas infra-renais e para-renais, apresenta índice de mortalidade e morbidade consideravel mente maior ${ }^{10}$. C itamse taxas de mortalidade para esse tipo de aneurisma em torno de 3,1 a 20\% em cirurgias eletivas, com uma taxa deisquemia medular em torno de 1,3 a 4,5\% eocorrência de insuficiência renal em 8,8 a $14 \%$ dos casos $^{4-12}$.

Em 1972, W alker introduziu o termo aneurisma inflamatório da aorta abdominal (embora James e D eW eerd terem descrito a doença em 1935 e 1955, respectivamente), em que há um espessamento esbranquiçado da parede do vaso e extensa fibroseenvolvendo ostecidosadjacentes ${ }^{4,13}$. Essesaneurismasaumentam 0 risco decomplicações duranteeapóso ato cirúrgico, em função das múltiplas aderências encontradas entre 0 duodeno $(100 \%)$, veia renal esquerda $(48-51 \%)$, veia cava inferior $(63-70 \%)$, ureteres $(20-44 \%)$, delgado $(20 \%)$ e sigmóide $(5-20 \%)^{10}$. São sintomáticos em $80 \%$ dos casos e apresentam incidência de 3 a 12\% entre aneurismas da aorta, raramente atingindo a aorta acima da emergência das artérias renais ${ }^{1,13}$. Prevalecem no sexo masculino (6-30:1), em pacientes mais jovens e fumantes, em relação aos aneurismas não-inflamatórios ${ }^{13}$. 
Entre 1957 e 1984, Crawford e Stowe trataram 30 pacientes com aneurisma inflamatório da aorta apenas seis $(20 \%)$ desses acometiam a aorta acima das artérias renais - erelataram apenas uma morte no período de 30 dias após a correção cirúrgica desses casos $^{3}$.

Arroyo encontrou $17,2 \%$ dos AT A tipo IV associados com aneurisma inflamatório, sendo que $10 \%$ dos seus aneurismas inflamatórios eram AT A, não havendo mortalidade cirúrgica nessa série ${ }^{4}$.

$N$ esse relato de caso, a tomografia evidenciava um aneurisma com início na emergência da artéria mesentérica superior, com sinais de espessamento da parede em toda sua circunferência, porém esse exame não demonstrou o duplo halo de contraste característico dos aneurismas inflamatórios, talvez devido à técnica utilizada (apenas 13-33\% desses aneurismas são diagnosticados no pré-operatório) ${ }^{11}$. $\mathrm{N}$ ão dispomos do VHS pré-operatório, nem da biópsia da parede da aorta, mas os achados intra-operatórios confirmaram a etiologiainflamatóriado aneurisma (Figura4) edificultaram sobremaneira a exposição da artéria renal esquerda e do colo distal do aneurisma. Proximalmente, a fibrosenão se estendia até o pilar diafragmático, mas as aderências distais acarretaram um sangramento difuso e contínuo do retroperitônio, exposto após a rotação medial das vísceras.

$\mathrm{N}$ ão realizamos, de rotina, ureterolise nos aneurismas inflamatórios, conforme preconizado por Crawford, Sterpetti e Rasmussen ${ }^{13}$, uma vez que ocorre regressão dafibrosequeenvolveosureteresem $70 \%$ dos casos e, raramente, há piora da função renal secundária ao "aprisionamento" ureteral pós-operatório ${ }^{13}$.

0 ptamos por usar a perfusão visceral com circuito idealizado em nosso serviço (Figuras 3 e 4) ${ }^{14}$, devido ao possível tempo de pinçamento prolongado nesse caso de múltiplas aderências e exposição limitada. Além disso, um dos indicadores pré-operatórios de mortalidade na cirurgia dos ATA é a creatinina acima de $1,5 \mathrm{mg} / \mathrm{dl}^{10}$, reforçando a necessidade de preservar a perfusão renal durante o pinçamento aórtico em pacientes com função renal anormal.

$\mathrm{N}$ a literatura, ainda é controverso o uso de perfusão visceral em AT A IV , sendo que muitos autores têm bom resultado com essa técnica ${ }^{15-17}$, enquanto que outros apresentam os mesmos resultados com 0 clamp and go ou apenas perfusão de solução cristalóide gelada7-9,18. Acreditamos que cada caso deve ser avaliado em particular, levando-se em consideração as dificuldades técnicas encontradas. A associação de um AT A com aneurisma inflamatório parece ser uma boa indicação da perfusão visceral durante o pinçamento da aorta supra-renal.

\section{R eferências}

1. Coselli JS. Contribution of E. Stanley Crawford in aortic thoracoabdominal aneurysms. In: Yao JS, Pearce WH. Aneurysms: new findings and treatments. Appleton \& Lange; 1995. p. 173-195.

2. Luccas GC, Prezotti M A, M atsui IA, M enezes FH. Anomalias venosas associadas à cirurgia de aorta abdominal. Cir Vasc Angiol. 1999;15:51-4.

3. Crawford JL, Stowe CL, Safi H J, H allman CH, Crawford ES. Inflammatory aneurysms of the aorta. J Vasc Surg. 1985;2: 113-24.

4. Arroyo A, Barrio C, Alvarez A, Carmona S, M ontero R, G esto $R$. Inflammatory aneurysm in the infrarenal portion of thoracoabdominal aneurysms: an uncommon variant. J V asc Surg. 2003;37:1006-8.

5. $\mathrm{M}$ artin $\mathrm{GH}, \mathrm{O}^{\prime} \mathrm{H}$ ara $\mathrm{PJ}, \mathrm{H}$ ertzer $\mathrm{NR}$, et al. Surgical repair of aneurysmsinvolving thesuprarenal, visceral, and lower thoracic aortic segments: early results and late outcome. J V asc Surg. 2000;31:851-62.

6. Schwartz LB, Belkin M, Donaldson MC, M annick JA, W hittemore $A D$. Improvement in results of repair of type IV thoracoabdominal aortic aneurysms. J ournal V ascular Surgery 1996 July;24(1): 74-81.

7. Schepens MA, D efauw JJ, Hamerlijnck RP, D e Geest R, $V$ ermeulen. FE. Surgical treatment of thoracoabdominal aortic aneurysms by simple crossclamping. J Thorac Cardiovasc Surg. 1994;107:134-42.

8. CambriaRP, D avison JK, Z annetti S, L'Italien G, Atamian S. Thoracoabdominal aneurysm repair. Ann Surg. 1997;226:294-305.

9. Coselli JS, LeM aire SA, M iller CC, et al. Mortality and paraplegia after thoracoabdominal aortic aneurysm repair: a risk factor analysis. Ann Thorac Surg. 2000;69:409-14.

10. CD Bicknell, AR Cowan, MI Kerle, AO Mansfield, NJ Cheshire, JH W olfe. Renal dysfunction and prolonged visceral ischaemia increase mortality rate after suprarenal aneurysm repair. Br J Surg. 2003;90:1142-6.

11. dos Santos VP, I gnácio M R, da Silveira DR, Caffaro RA. Aneurisma toracoabdominal roto: relato de um caso com uso de anel rígido sulcado de $D$ elrin intraluminal na anastomose proximal. J Vasc Br. 2004;3:383-6.

12. Anacleto $A$, Anacleto JC.Aneurismas da aorta torácica e toracoabdominal. In: deB rito CJ. Cirurgiavascular. 1 a ed. R io de Janeiro: Revinter; 2002. p. 439.

13. TE R asmussen, JW H allett. Inflammatory aortic aneurysms: a clinical review with new perspectives in pathogenesis. Ann Surg. 1997;225:155-64.

14. RochaEF, G uillaumon AT, AntunesN , VieiraRW . Aneurisma toracoabdominal roto: modificação do circuito de perfusão visceral. Rev Bras Cir Cardiovasc. 2004;19:413-16. 
15. Kuniyoshi $Y$, Koja K, M iyagi $K$, et al. Selective visceral perfusion during thoracoabdominal aortic aneurysm repair. Ann Thoracic Cardiovasc Surg. 2004;10:367-72.

16. M orishita K, Yokoyama H, Inoue S, Koshino T, T amiya $Y$, Abe T. Selective visceral and renal perfusion in thoracoabdominal aneurysm repair. Eur J C ardiothorac Surg. 1999; 5:502-7.

17. Jacobs MJ, Eijsman L, M eylaerts SA, et al. Reduced renal failure following thoracoabdominal aortic aneurysm repair by selectiveperfusion. Eur J Cardiothorac Surg. 1998;14:201-5.

18. EideT O, Romundstad P, Saether O D, M yhreH O, Aadahl P. A strategy for treatment of type III and IV thoracoabdominal aortic aneurysm. Ann V asc Surg. 2004;18:408-13.

\section{Correspondência:}

Eduardo Faccini Rocha

Rua C ônego Januário Barbosa, 427

CEP $18030-200$ - Sorocaba, SP

Tel.: (15) 3231.6189

E-mail: eduardofaccinirocha@bol.com.br 